\title{
Electromagnetic Modeling of Dielectric Mixtures
}

\author{
Luigi La Spada*, Renato lovine and Lucio Vegni
}

\author{
Department of Engineering, University of Roma Tre, Via Vito Volterra 62, 00146, Rome, Italy
}

\begin{abstract}
Electromagnetic modeling of dielectric materials allows us to study the effects of electromagnetic wave propagation and how such electromagnetic fields influence and interact with them.

Dielectric materials are composites or mixtures, which often are made up of at least two constituents or phases. Modelling the electromagnetic behaviour of dielectric mixtures is crucial to understand how geometrical factors (shape and concentration), electromagnetic properties of inclusions and background medium, influence the permittivity of the overall material.

The aim of this work is to develop new analytical models for dielectric mixtures, in order to describe their electromagnetic behaviour and design them with desired electromagnetic properties, for specific required applications. In particular, in this paper a new general expression for the effective permittivity of dielectric mixture is presented. The mixtures consist of inclusions, with arbitrary shapes, embedded in a surrounding dielectric environment. We consider the hosting environment and the hosted material as real dielectrics, both of them as dispersive dielectrics.
\end{abstract}

The proposed analytical models simplify practical design tasks for dielectric mixtures and allow us to understand their physical phenomena and electromagnetic behaviours.

Keywords: Analytical models, dielectric mixtures, effective permittivity, dispersive models, polymers.

\section{INTRODUCTION}

Dielectric mixtures are of great scientific interest due to their potential role in several application fields such as medicine, optics and electronics [1].

Their particular electromagnetic properties make them suitable for several applications involving optical and photonic ones [2], biochemical sensing and detection $[3,4]$, protein analysis [5,6], cell membrane function [7], biomedical applications [8,9], food quality analysis [10] and imaging [11,12], heating therapy techniques, in vivo tumour cell targeting and early diagnostic techniques [13].

A great interest in dielectric mixtures is the development of new analytical models, describing their electromagnetic behaviours.

Mathematical modelling turns out to be a useful tool in order to study their physical properties, how such structures influence and interact with the surrounding environment, how to manipulate, control and design their electromagnetic properties for specific required applications.

Therefore, the aim of this paper is to present new design methods for dielectric mixtures with desired electromagnetic properties. The findings of this study help us to understand their electromagnetic behaviour

*Address correspondence to this author at the Department of Engineering, University of Roma Tre, Via Vito Volterra 62, 00146, Rome, Italy; Tel: +39.06.57337065; Fax: +39.06.57337003; E-mail: luigi.laspada@uniroma3.it and give us the possibility to use them for materials electromagnetic modelling.

In particular the article is focused on the evaluation of the electromagnetic properties of arbitrary shape inclusion dielectric mixture in terms of extinction cross section (absorption and scattering) and effective permittivity. For this purpose, new analytical closedform formulas, linking the geometrical and the electromagnetic parameters of such structures with their resonant frequency properties, are evaluated.

The capability to manipulate and control the electromagnetic phenomenon, by exploiting the proposed analytical models, opens up several possible applications. In fact, the main advantage of using the analytical models, proposed in this paper, give us the possibility to tune the structure resonant properties (in terms of position, amplitude and bandwidth) by changing its geometrical and electromagnetic characteristics, for specific required applications.

\section{ANALYTICAL MODELS AND ELECTRO- MAGNETIC PROPERTIES OF A DIELECTRIC MIXTURE}

In this section, the general analytical expression of the structure effective permittivity is derived and the dependence on its geometry, its electromagnetic properties, and the permittivity of the surrounding dielectric environment, is presented. The proposed structures consist of resonant arbitrary shape inclusions, arranged in an array configuration, whose frequency response is modified through the refractive 


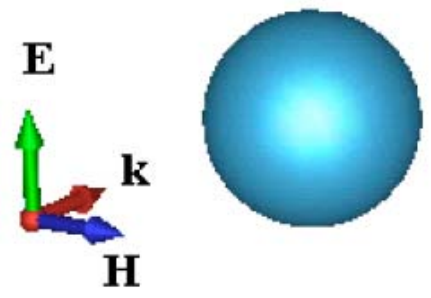

a

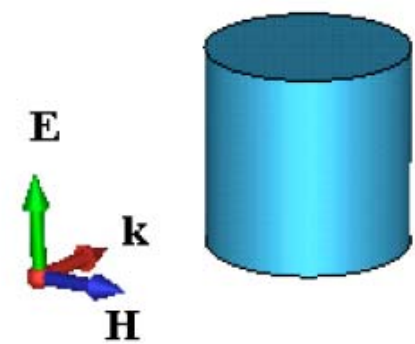

b

Figure 1: Examples of dielectric inclusions geometry.

index variation of the surrounding dielectric environment. Let us assume that the structure is excited by an impinging electromagnetic plane wave, having the electric field $\mathbf{E}$ parallel and the propagation vector $\mathbf{k}$ perpendicular to the inclusion principal axis, respectively, as shown in Figure 1. The dielectric mixture electromagnetic properties are revealed in terms of extinction cross-section. The mixture holds specific resonant frequency properties in terms of position, magnitude and bandwidth, depending on its geometrical (shape, dimensions) and on the electromagnetic characteristics of the inclusion it is made of. In order to study the structure electromagnetic properties, the following assumptions must be done:

Inclusion are considered "electrically small": their sizes are much smaller than the operative wavelength in the surrounding medium [14]. As a consequence the resonant behaviour can be studied in terms of a quasistatic approximation;

Particles and the surrounding dielectric environment are considered homogeneous.

To describe the structure electromagnetic behavior it is necessary to evaluate their electromagnetic extinction cross section properties, in terms of scattering and absorption.

Separated evaluation of both phenomena is crucial to understand why certain structures are preferred to others for specific applications. By evaluating analytical formulas for extinction cross-section properties, it is possible to correlate the entire structure electromagnetic properties with its geometrical characteristics, in order to describe its resonant behaviour, in terms of wavelength position, magnitude and bandwidth. Apart from geometry such as sphere and cylinder [15], cube [16], disc [17] and needle [18], whose closed form formulas are noted in literature, for inclusions of arbitrary shapes the electromagnetic solution is obtained by numerical approaches [19].
The corresponding expressions, linking the inclusion polarizability $\alpha$ to the structure extinction properties, for absorption $\left(\mathrm{C}_{\mathrm{abs}}\right)$ and scattering $\left(\mathrm{C}_{\mathrm{sca}}\right)$ cross-section read, respectively [18]:

$$
\begin{aligned}
& \mathrm{C}_{\mathrm{abs}}=\mathrm{k} \operatorname{Im}[\alpha] \\
& \mathrm{C}_{\mathrm{sca}}=\frac{\mathrm{k}^{4}}{6 \pi}|\alpha|^{2}
\end{aligned}
$$

where $k=(2 \pi n) / \lambda$ is the wave-number, $\lambda$ is the wavelength, $n$ is the refractive index of the surrounding dielectric environment and $\alpha$ the particle polarizability.

Under such conditions it is possible to correlate the structure extinction resonant electromagnetic properties with the inclusion polarizability expression.

In the limit of the quasi-static approximation, in case of an arbitrary shaped particle, the scalar component of the dyadic polarizability along one of its geometrical axes, can be expressed as [20]:

$\alpha=\mathrm{V} \varepsilon_{\mathrm{e}} \frac{\varepsilon_{\mathrm{i}}-\varepsilon_{\mathrm{e}}}{\varepsilon_{\mathrm{e}}+\mathrm{L}_{\mathrm{x}, \mathrm{y}, \mathrm{z}}\left(\varepsilon_{\mathrm{i}}-\varepsilon_{\mathrm{e}}\right)}$

Where $V$ is the particle volume, $\varepsilon_{e}$ is the surrounding dielectric environment permittivity, $\varepsilon_{i}$ is the inclusion dielectric permittivity and $L$ is the depolarization factor.

Following the same procedure in [20], the new correspondent general expression of the effective permittivity for an arbitrary shape particle embedded in a dielectric environment reads:

$$
\varepsilon_{\text {eff }}=\frac{\varepsilon_{\mathrm{e}}\left((\mathrm{f}-1) \varepsilon_{\mathrm{e}}\left(\mathrm{L}_{\mathrm{x}, \mathrm{y}, \mathrm{z}}-1\right)+\varepsilon_{\mathrm{i}}\left(\mathrm{f}-(\mathrm{f}-1) \mathrm{L}_{\mathrm{x}, \mathrm{y}, \mathrm{z}}\right)\right)}{\varepsilon_{\mathrm{e}}\left((\mathrm{f}-1) \mathrm{L}_{\mathrm{x}, \mathrm{y}, \mathrm{z}}+1\right)-(\mathrm{f}-1) \varepsilon_{\mathrm{i}} \mathrm{L}_{\mathrm{x}, \mathrm{y}, \mathrm{z}}}
$$

where $f$ is the volume fraction of the inclusions in the mixture. 
In this paper we consider the hosting environment and the hosted material as real dielectrics. The following case studies are considered:

1. The inclusion is assumed to be a dispersive and homogeneous dielectric, instead the environment a dispersionless and lossless material.

2. The inclusion and the environment are both considered as dispersive and homogeneous dielectrics.

It will be shown that in the both considered cases, under specific conditions, the mixture effective permittivity follows a general Lorentz-like model.

\subsection{Inclusions with Lorentz Dispersion Model in a Dispersionless and Lossless Environment}

Starting from (3), as inclusions and environment permittivity can be expressed as $\varepsilon_{\mathrm{i}}(\omega)=\varepsilon_{\infty 1}+\frac{\omega_{\mathrm{p} 1}^{2}}{\omega_{01}^{2}-\omega^{2}-\mathrm{j} \gamma_{1} \omega}$ and $\varepsilon_{\mathrm{e}}$ respectively, the total effective permittivity expression reads as follow:

$$
\varepsilon_{\text {eff }}(\omega)=\frac{\varepsilon_{\mathrm{e}}\left(\begin{array}{l}
\omega_{\mathrm{p} 1}^{2}\left((\mathrm{f}-1) \mathrm{L}_{\mathrm{x}, \mathrm{y}, \mathrm{z}}-\mathrm{f}\right)+\left(\omega^{2}+\mathrm{j} \gamma_{1} \omega-\omega_{01}^{2}\right) \\
\left((\mathrm{f}-1)\left(\varepsilon_{\mathrm{e}}-1\right) \mathrm{L}_{\mathrm{x}, \mathrm{y}, \mathrm{z}}-(\mathrm{f}-1) \varepsilon_{\mathrm{e}}+\mathrm{f}\right)
\end{array}\right)}{(\mathrm{f}-1) \mathrm{L}_{\mathrm{x}, \mathrm{y}, \mathrm{z}} \omega_{\mathrm{p} 1}^{2}+\left(\omega^{2}+\mathrm{j} \gamma_{1} \omega-\omega_{01}^{2}\right)}
$$

being $\omega_{p}=2 \pi f_{p}$ the plasma frequency, $\omega_{0}=2 \pi f_{0}$ the natural frequency, $\gamma$ the damping frequency, $\omega=2 \pi f$ the angular frequency and $\varepsilon_{\infty}$ the dielectric constant at high frequencies.

An example, in the $\mathrm{GHz}$ regime, for the effective permittivity in its real and imaginary part is reported in Figure 2, where the effects of the volume fraction $f$, the depolarization factor $L$ and the background permittivity

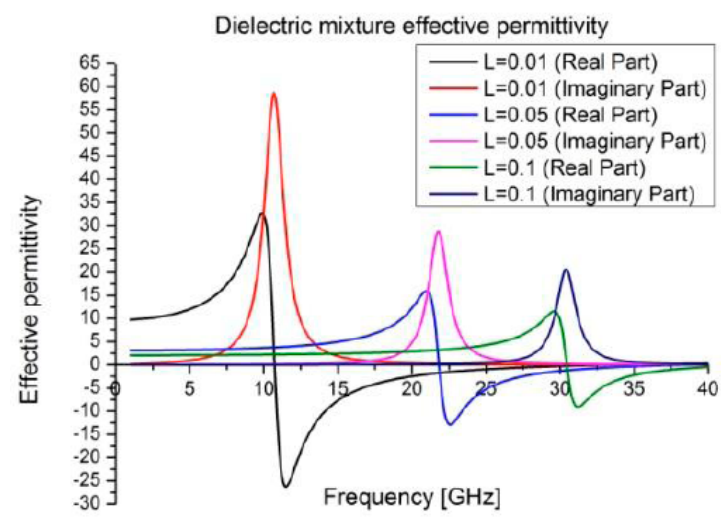

a

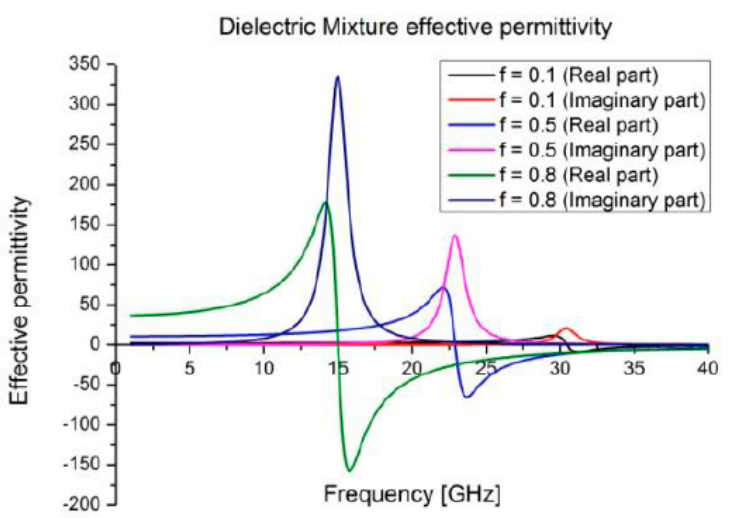

b

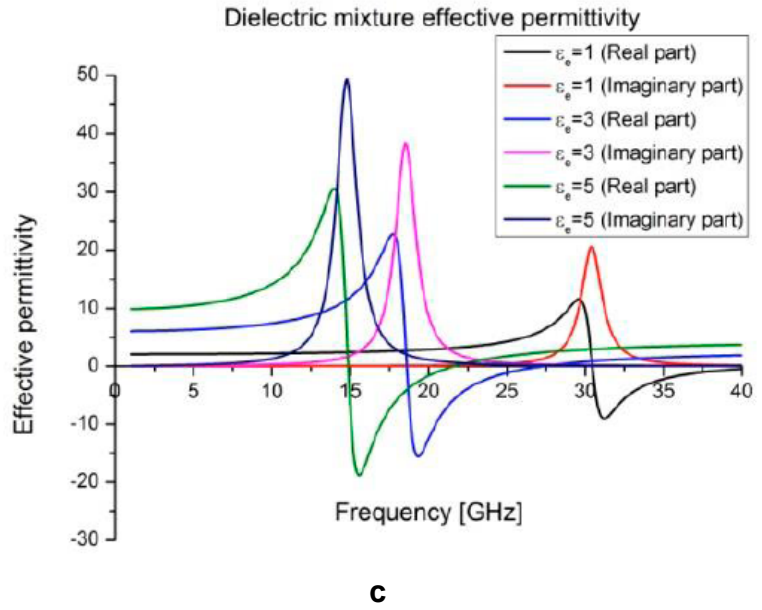

Figure 2: Variation of the dielectric mixture effective permittivity as a function of (a) depolarization factor $L\left(\varepsilon_{\mathrm{e}}=1 ; \varepsilon_{\infty}=1\right.$; $\left.\omega_{p 1}=2 \cdot \pi \cdot 100 \cdot 10^{9} \mathrm{THz} ; \omega_{01}=2 \cdot \pi \cdot 5 \cdot 10^{9} \mathrm{THz} ; \gamma_{1}=10 \cdot 10^{9} \mathrm{THz} ; \mathrm{f}=0.1\right) ;(\mathbf{b})$ volume fraction $\mathrm{f}\left(\varepsilon_{\mathrm{e}}=1 ; \varepsilon_{\infty}=1 ; \omega_{p 1}=2 \cdot \pi \cdot 100 \cdot 10^{9} \mathrm{THz} ;\right.$ $\left.\omega_{01}=2 \cdot \pi \cdot 5 \cdot 10^{9} \mathrm{THz} ; Y_{1}=10 \cdot 10^{9} \mathrm{THz} ; L=0.05\right)$ and $(\mathbf{c})$ surrounding dielectric permittivity $\varepsilon_{\mathrm{e}}\left(\varepsilon_{\infty}=1 ; \omega_{\mathrm{p} 1}=2 \cdot \pi \cdot 100 \cdot 10^{9} \mathrm{THz}\right.$; $\left.\omega_{01}=2 \cdot \pi \cdot 5 \cdot 10^{9} \mathrm{THz} ; \mathrm{V}_{1}=10 \cdot 10^{9} \mathrm{THz} ; \mathrm{f}=0.1 ; \mathrm{L}=0.05\right)$. 
are shown. As expected, only one resonant frequency is reached.

\subsection{Inclusions and Environment with Lorentz Dispersion Model}

Following the same procedure in paragraph 2.1, if inclusions and environment permittivity are expressed as

$$
\varepsilon_{\mathrm{i}}(\omega)=\varepsilon_{\infty 1}+\frac{\omega_{\mathrm{p} 1}^{2}}{\omega_{01}^{2}-\omega^{2}-\mathrm{j} \gamma_{1} \omega}
$$

and

$\varepsilon_{\mathrm{e}}=\varepsilon_{\mathrm{e}}(\omega)=\varepsilon_{\infty 2}+\frac{\omega_{\mathrm{p} 2}^{2}}{\omega_{02}^{2}-\omega^{2}-\mathrm{j} \gamma_{2} \omega} \quad$ respectively, the total effective permittivity expression, in terms of real and imaginary part, reads as follow:

$\varepsilon_{\text {eff }}(\omega)=\frac{\left(\omega^{2}-\omega_{\mathrm{p} 2}^{2}+\mathrm{j} \gamma_{2} \omega-\omega_{02}^{2}\right)\left(\mathrm{g}_{\mathrm{i}}(\omega)-\mathrm{g}_{\mathrm{e}}(\omega)+1\right)}{\mathrm{g}_{\mathrm{ie}}(\omega)+\omega_{\mathrm{p} 2}^{2}\left(-(\mathrm{f}-1) \mathrm{L}_{\mathrm{x}, \mathrm{y}, \mathrm{z}}-1\right)+\omega^{2}+\mathrm{j} \gamma_{2} \omega-\omega_{02}^{2}}$

with

$$
\begin{aligned}
& g_{i}(\omega)=\frac{\omega_{p 1}^{2}\left((f-1) L_{x, y, z}-f\right)}{\omega^{2}+j \gamma_{1} \omega-\omega_{01}^{2}} \\
& g_{e}(\omega)=\frac{(f-1)\left(L_{x, y, z}-1\right) \omega_{p 2}^{2}}{\omega^{2}+i \gamma_{2} \omega-\omega_{02}^{2}} \\
& g_{i e}(\omega)=\frac{(f-1) L_{x, y, z} \omega_{p 1}^{2}\left(\omega^{2}+j \gamma_{2} \omega-\omega_{02}^{2}\right)}{\omega^{2}+j \gamma_{1} \omega-\omega_{01}^{2}}
\end{aligned}
$$

An example for the effective permittivity in its real and imaginary part is reported in Figure 3 . In this case, by using dispersion in both materials two resonant frequencies are possible.

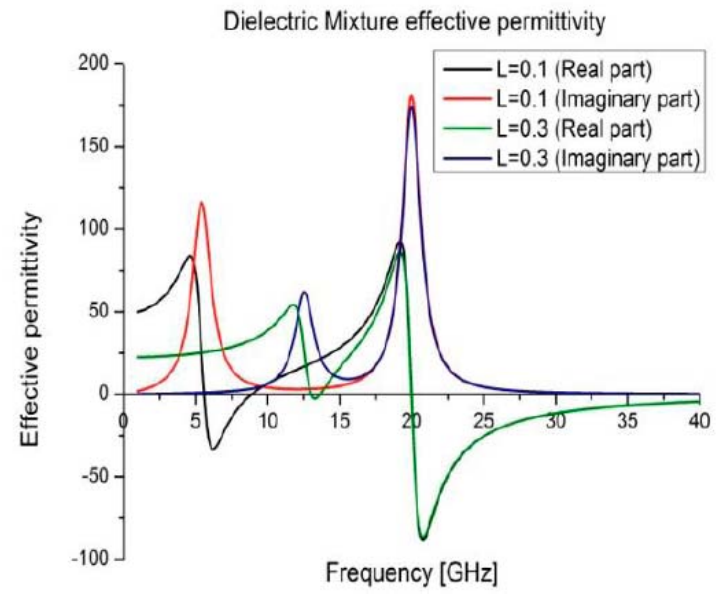

a

\section{MIXTURES ELECTROMAGNETIC MODELING}

In this section the design of a dielectric mixture for modelling applications is proposed. As it will be shown by analytical results, the proposed structure can be used as a powerful tool for a reliable model design for polymers or mixtures of them in the infrared (IR) region. In particular, in order to test our modelling approach, we referee to a specific polymer largely used in electromagnetism: the Poly-Methyl MethAcrylate (PMMA).

PMMA is a strong and lightweight material. It transmits up to $92 \%$ of the visible light and gives a reflection of about $4 \%$. It is also a good filter for ultraviolet light at wavelengths below about $300 \mathrm{~nm}$. For what concern the IR spectrum, PMMA passes infrared light up to $2800 \mathrm{~nm}$ and blocks IR of longer wavelengths up to $25000 \mathrm{~nm}$. Such peculiar electromagnetic properties make such material suitable for several applications: for instance to build-up heat sensors.

The dielectric properties of PMMA result from the interaction of electromagnetic radiation with its constituents at the molecular level. These variations imply significant changes in their electromagnetic properties.

The IR spectrum of the PMMA reflects the presence of the $\mathrm{C}-\mathrm{O}$ bonds within the polymer. Such bonds dominate the majority of the bending and stretching vibration peaks present in the material [21]. To describe the optical properties of PMMA, the DrudeLorentz model $[22,23]$ is used, which defines the electric permittivity as follows:

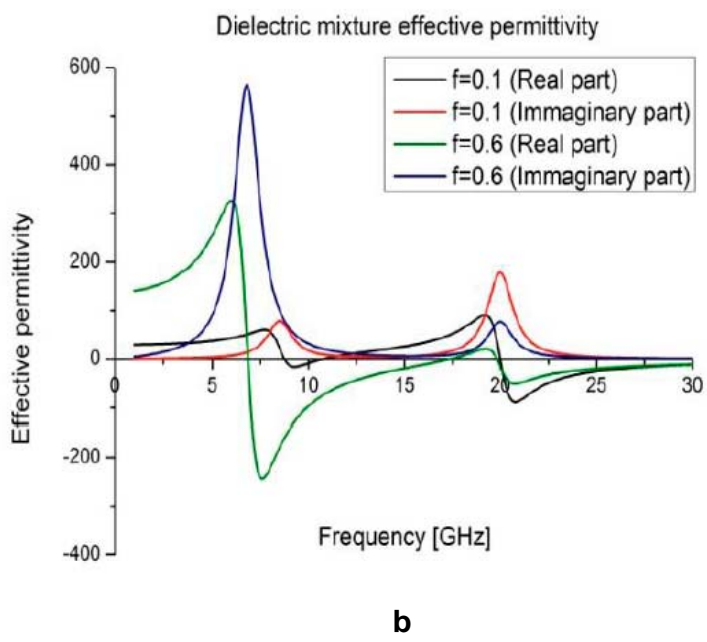

Figure 3: Variation of the dielectric mixture effective permittivity as a function of $(\mathbf{a})$ depolarization factor $L\left(\varepsilon_{\mathrm{e}}=10 ; \varepsilon_{\infty}=1\right.$; $\left.\omega_{p 1}=2 \cdot \pi \cdot 100 \cdot 10^{9} \mathrm{THz} ; \omega_{01}=2 \cdot \pi \cdot 5 \cdot 10^{9} \mathrm{THz} ; \gamma_{1}=10 \cdot 10^{9} \mathrm{THz} ; \omega_{\mathrm{p} 2}=2 \cdot \pi \cdot 80 \cdot 10^{9} \mathrm{THz} ; \omega_{02}=2 \cdot \pi \cdot 20 \cdot 10^{9} \mathrm{THz} ; \mathrm{Y}_{2}=10 \cdot 10^{9} \mathrm{THz} ; \mathrm{f}=0.1\right)$ and (b) volume fraction $f\left(\varepsilon_{\mathrm{e}}=10 ; \quad \varepsilon_{\infty 1}=1 ; \quad \omega_{\mathrm{p} 1}=2 \cdot \pi \cdot 100 \cdot 10^{9} \mathrm{THz} ; \omega_{01}=2 \cdot \pi \cdot 5 \cdot 10^{9} \mathrm{THz} ; \quad \gamma_{1}=10 \cdot 10^{9} \mathrm{THz} ; \omega_{\mathrm{p} 2}=2 \cdot \pi \cdot 80 \cdot 10^{9} \mathrm{THz} ;\right.$ $\left.\omega_{02}=2 \cdot \pi \cdot 20 \cdot 10^{9} \mathrm{THz} ; \gamma_{2}=10 \cdot 10^{9} \mathrm{THz} ; \mathrm{L}=0.1\right)$. 
$\varepsilon(\omega)=\varepsilon_{\infty}+\sum_{\mathrm{k}} \frac{\omega_{\mathrm{pk}}^{2}}{\omega_{0 \mathrm{k}}^{2}-\omega^{2}-\mathrm{j} \gamma_{\mathrm{i}} \omega}$

Each absorption frequency can be related to the electromagnetic behaviour of a damped harmonic oscillator. Therefore the (7) describes the optical response of a set of such oscillators. In this formula, $\varepsilon_{\infty}$ is the high-frequency dielectric constant, which represents the contribution of all oscillators at very high frequencies, compared to the frequency range under study. The parameters $\omega_{\mathrm{pk}}, \omega_{0 \mathrm{k}}$ and $\gamma_{\mathrm{k}}$, are the "plasma" frequency, the resonant frequency and damping frequency, respectively, of the k-th Lorentz oscillator.

The main purpose is to show how to replicate the dispersive electromagnetic properties of PMMA by using the aforementioned proposed electromagnetic modelling approach.

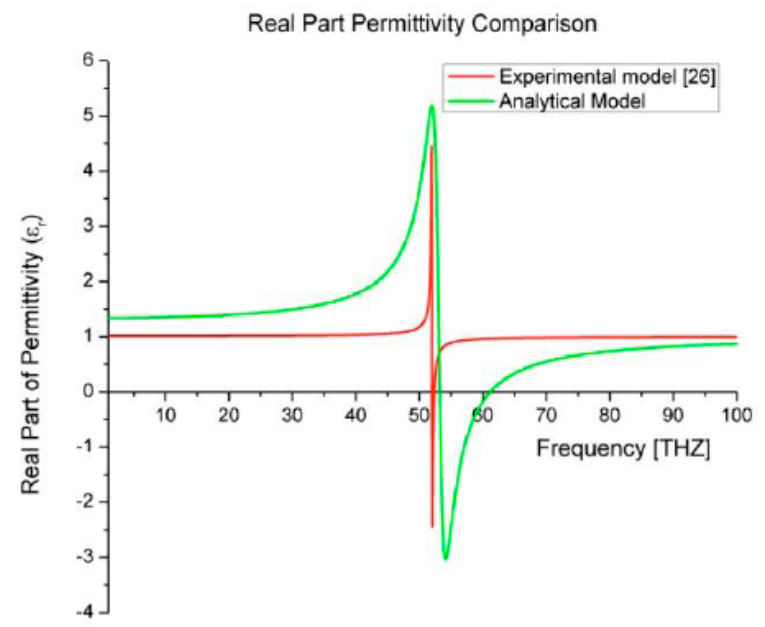

a

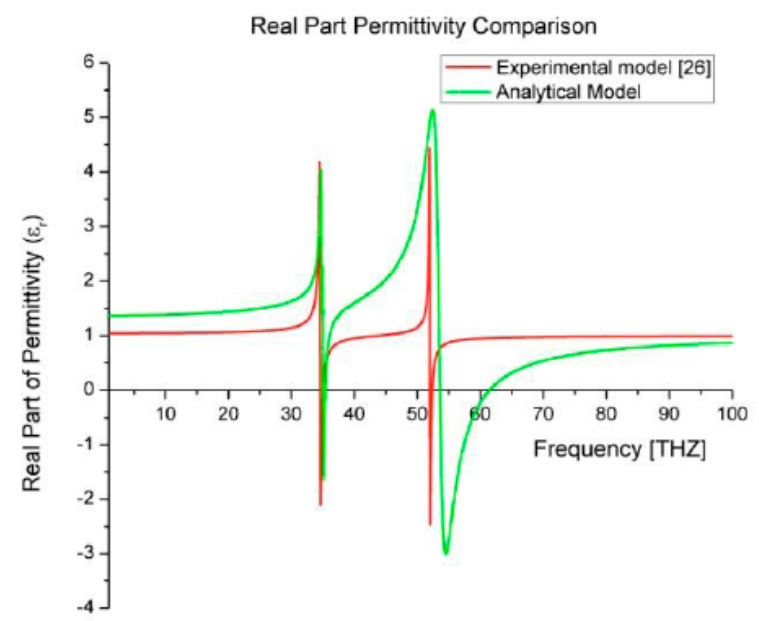

In particular in this work, we have obtained that:

- $\quad$ For the single resonant frequency structure the mixture has to be characterized by the following geometrical parameters: $L=0.03, f=0.01$, and by the following inclusion material properties [24]: $\omega_{01}=197 \mathrm{THz}, \mathrm{y}_{01}=11.6 \mathrm{THz}, \omega_{\mathrm{p} 1}=2178$ $\mathrm{THz}, \varepsilon_{\mathrm{e}}=2.08$. The mixture is composed by gold inclusions embedded in a glass substrate.

- For the multi-resonant frequency structure the mixture has to be characterized by the following geometrical parameters: $L=0.028, f=0.01$. The inclusion and environment material properties have to be [25]: $\omega_{01}=197 \mathrm{THz}, \mathrm{Y}_{01}=11.6 \mathrm{THz}$, $\omega_{\mathrm{p} 1}=2178 \mathrm{THz}, \omega_{02}=2 \pi \cdot 35 \mathrm{THz}, \mathrm{Y}_{02}=1 \mathrm{THz}$, $\omega_{\mathrm{p} 2}=2 \pi \cdot 5 \mathrm{THz}$. In this case, the mixture can be composed by inclusions depicted in Figure $\mathbf{1 b}$.
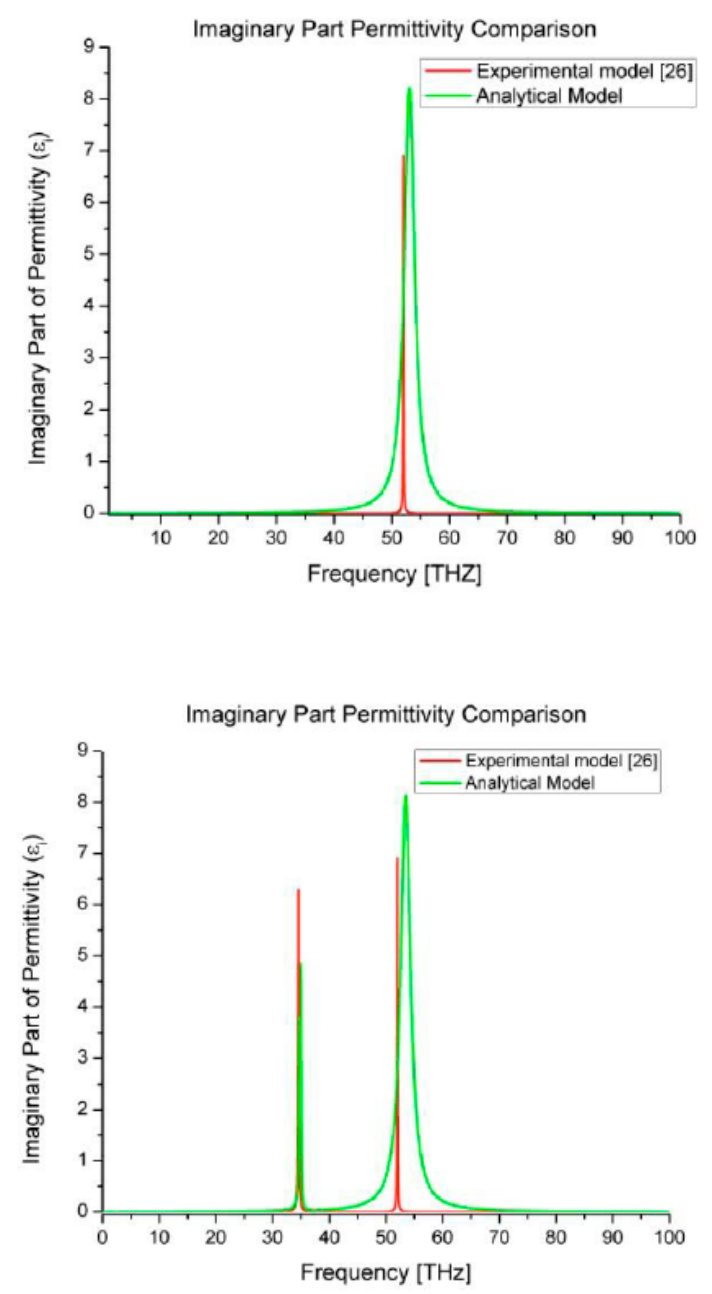

b

Figure 4: Modeling of PMMA dispersion behavior with: (a) a single resonant structure, (b) a multi resonant structure. 
- To describe the dielectric behavior of the PMMA, the absorbance models presented in [26] have been used.

The obtained results, for both structures, are shown in Figure $\mathbf{4 a}$ and $\mathbf{4 b}$, respectively.

The most remarkable result to emerge from Figure 4 for both structure, is that a good agreement is reached between the experimental results existing in literature [26], and the proposed modeling approach. Our formulation reproduces pretty well the electromagnetic response of the PMMA polymer. This concept can be extended to other polymers and in particular to other frequency ranges such as microwave, millimeter-wave and optical frequencies.

\section{CONCLUSIONS}

This work is focused on the study of dielectric mixture properties, in particular how to manipulate and control their electromagnetic properties. In this paper, a study on metamaterials electromagnetic modelling and on its main applications, operating in the infrared frequency regime, is presented. The proposed analytical models simplify practical design tasks and allow us a deeper understanding of physical phenomena of such structures. The possibility to predict the electromagnetic behaviour of different kind of structures is of considerable interest to build up mixtures that satisfy desired requirements. The main advantage of using such models is the possibility to tune their resonance properties by changing their geometrical and electromagnetic characteristics, for specific required applications. Therefore, by using the proposed formulas, a new dielectric mixture simulating the polymer electromagnetic behaviour of PMMA in the infrared frequency range has been designed.

\section{REFERENCES}

[1] Pendry JB. Playing Tricks with Light. Science 1999; 285: 1687-88.

http://dx.doi.org/10.1126/science.285.5434.1687

[2] Chau YF, Jiang ZH, Li HY, Lin GM, Wu FL, Lin WH. Localized resonance of composite core-shell nanospheres, nanobars and nanospherical chains. Progr Electromagnet Res 2011; 28: 183-99.

[3] Maier SA, Kik PG, Atwater HA, Meltzer S, Harel E, Koel BE, Requicha $A G$. Local detection of electromagnetic energy transport below the diffraction limit in metal nanoparticle plasmon waveguides. Nat Mater 2003; 2: 229-32. http://dx.doi.org/10.1038/nmat852

[4] Riboh JC, Haes AJ, McFarland AD, Ranjit C, Van Duyne RP. A Nanoscale Optical Biosensor: Real Time Immunoassay and Nanoparticle Adhesion. J Phys Chem B 2003; 107: 1772-80.

http://dx.doi.org/10.1021/jp022130v
[5] Bukasov R, Ali TA, Nordlander P, Shumaker-Parry JS. Probing the plasmonic near-field of gold nanocrescent antennas. ACS Nano 2010; 4: 6639-50. http://dx.doi.org/10.1021/nn101994t

[6] Storhoff JJ, Elghanian R, Mucic RC, Mirkin CA, Letsinger RL. DNA Directed Synthesis of Binary Nanoparticle Network Materials. J Am Chem Soc 1998; 120: 12674-5. http://dx.doi.org/10.1021/ja982721s

[7] Larsson EM, Alegret J, Kall M, Sutherland DS. Sensing characteristics of NIR localized surface plasmon resonances in gold nanoring for application as ultrasensitive biosensors. Nano Lett 2007; 7: 1256-63. http://dx.doi.org/10.1021/nl0701612

[8] Galush WJ, Shelby SA, Mulvihill MJ, Tao A, Yang P, Groves JT. A nanocube plasmonic sensor for molecular binding on membrane surfaces. Nano Lett 2009; 9: 2077-82. http://dx.doi.org/10.1021/nl900513k

[9] Rosi NL, Mirkin CA. Nanostructures in biodiagnostics. Chem Rev 2005; 105: 1547-62. http://dx.doi.org/10.1021/cr030067f

[10] Chen J, Wiley BJ, Cang H, Campbell D, Saeki F, Au L, et al. Gold Nanocages: Engineering the Structures for Biomedical Applications. Adv Mater 2005; 17: 2255-61. http://dx.doi.org/10.1002/adma.200500833

[11] Hiep HM, Endo T, Kerman K, Chikae M, Kim DK, Yamamura $\mathrm{S}$, et al. A localized surface plasmon resonance based immunosensor for the detection of casein in milk. Sci Technol Adv Mater 2007; 8: 331-38. http://dx.doi.org/10.1016/j.stam.2006.12.010

[12] Yelin D, Oron D, Thiberge S, Moses E, Silberberg Y, Willner I. Multiphoton plasmon-resonance microscopy. Opt Express 2003; 11: 1385-91 http://dx.doi.org/10.1364/OE.11.001385

[13] Sokolov K, Follen M, Aaron J, Pavlova I, Malpica A, Lotan R, Richards-Kortum R. Real-Time Vital Optical Imaging of Precancer Using Anti-Epidermal Growth Factor Receptor Antibodies Conjugated to Gold Nanoparticles. Cancer Res 2003; 63: 1999-2004.

[14] Bohren C, Huffmann D. Absorption and Scattering of Light by Small Particles. New-York, John Wiley 1983.

[15] Yaghjian AD. Electric dyadic Green's functions in the source region. Proc IEEE 1980; 68: 248-63.

\section{http://dx.doi.org/10.1109/PROC.1980.11620}

[16] Avelin J, Arslan AN, Brännback J, Flykt M, Icheln C Juntunen $\mathrm{J}$, et al. Electric fields in the source region: the depolarization dyadic for a cubic cavity. Electr Eng 1998; 81 : 199-202. http://dx.doi.org/10.1007/BF01233270

[17] Landau LD, Lifshitz EM. Electrodynamics of Continuous Media. Oxford, Pergamon Press 1984

[18] Van Bladel JG. Electromagnetic Fields. Hoboken, John Wiley \& Sons 2007 http://dx.doi.org/10.1002/047012458X

[19] Sihvola A. Dielectric Polarization and Particle Shape Effects. J Nanomater 2007; 1: 1-9. http://dx.doi.org/10.1155/2007/45090

[20] Sihvola A. Electromagnetic Mixing Formulas and Applications. London, The Institution of Engineering and Technology 2008.

[21] Kasarova SN, Sultanova NG, Ivanov CD, Nikolov ID. Analysis of the dispersion of optical plastic materials. Optic Mater 2007; 29: 1481-90. http://dx.doi.org/10.1016/j.optmat.2006.07.010

[22] Kuzmenko AB. Kramers-Kronig constrained variational analysis of optical spectra. Rev Sci Instrum 2005; 76 083108.1-9.

[23] Yamamoto K. Optical theory applied to infrared spectroscopy. Vib Spectros 1994; 8: 1-36. http://dx.doi.org/10.1016/0924-2031(94)00022-9 
[24] Rakic AD, Djurisic AB, Elazar JM, Majewski ML. Optical properties of metallic films for vertical-cavity optoelectronic devices. Appl Opt 1998; 37: 5271-83. http://dx.doi.org/10.1364/AO.37.005271

[25] Blythe AR. Electrical Properties Of Polymers. Cambridge, Cambridge University Press 2005.
[26] Jitian S, Bratu I. Determination of optical constants of polymethyl methacrylate films from IR reflection-absorption spectra. Proceedings of AIP Conferences 2012; 1425: 26-29. http://dx.doi.org/10.1063/1.3681958

Received on 01-10-2013

Accepted on 23-12-2013

Published on 13-01-2014

DOI: http://dx.doi.org/10.6000/1929-5995.2013.02.04.2

(C) 2013 La Spada et al.; Licensee Lifescience Global.

This is an open access article licensed under the terms of the Creative Commons Attribution Non-Commercial License (http://creativecommons.org/licenses/by-nc/3.0/) which permits unrestricted, non-commercial use, distribution and reproduction in any medium, provided the work is properly cited. 